\title{
Sound Transmission Testing of Polymer Compounds
}

\author{
P S Allan, A Ahmadnia, R Withnall and J Silver \\ Wolfson Centre for Materials Processing, Brunel University, Uxbridge, Middlesex, \\ UB8 $3 \mathrm{PH}, \mathrm{UK}$
}

\begin{abstract}
Acoustic properties of polymer compounds are an important consideration for many applications. Currently there are standard test methods for the determination of these properties. There is, however, no standard for the equipment used in these tests. There is only a specification for the test conditions. The objective of this work was to evaluate the operation and performance of a bench top laboratory sound testing system for its potential as a simple cost effective method for the initial evaluation of materials that require specific acoustic properties. The work was limited to an investigation of the property of sound transmission loss (STL). A study of the effect of the mounting conditions for the samples on the STL was carried out. Following this a series of polymer and polymer composite samples was tested. The results presented demonstrate the potential for the testing system as an effective standard test method for the acoustic properties of polymer composites and other materials.
\end{abstract}

\section{Introduction}

Among the many applications of polymers and polymer compounds there are a significant number, particularly in the automotive, aerospace and construction industries that require low acoustic transmission properties. Generally the mechanical and physical properties of polymer based materials are well documented and understood from data taken by standard testing methods, but there is no or little information about their acoustic transmission properties. Due to demand for a more comfortable environment and also through regulatory pressures, there is a need to reduce the noise level in many manufactured items. A low sound transmission property is, therefore, an important consideration in the materials selection for these applications. Consequently, there is a requirement for a test method for the measurement of the sound transmission loss (STL) of materials. Unfortunately however, the current the test methods used for STL can be very involved and costly. For example, the standard test methods for measuring the STL of building materials typically involves the construction of two adjacent rooms in a large laboratory with the test materials installed to match a real situation. The sound is generated in one room, and measurements are taken in both the source and receiver room to characterize STL [1]. These methods are well defined, time tested, and reliable but are, very time consuming and expensive and could require large samples of the prototype material for each test. In the situations where many STL tests are necessary this cost and space burden is significant. A STL testing procedure that is less costly and requires less space would, therefore, be of great value for the development of materials with low acoustic transmission. An impedance tube apparatus has been used by researchers [1-4] to measure the acoustic properties of materials. This method has been standardized for the measurement of absorption coefficient and impedance by the procedures ASTM E1050-98 of the American Society for Testing and Materials (ASTM) and ISO 10534-2 of the International Standard Organisation (ISO) published in $1998[5,6]$ (ASTM E1050-98 has now been revised in the latest version E1050-10 [7]). More recently ASTM published a standard test procedure E2611-09 [8] for the measurement of sound transmission loss (STL) that was based on the sound tube apparatus described in their E1050-98 standard. This standard procedure describes 
a testing method for STL that could readily be carried out on a laboratory bench and therefore offers a cost effective way of evaluating materials that are being developed for applications with required sound transmission properties. Sound tube testing equipment that conforms to the above standard procedures is currently being manufactured by Bruel \& Kjaer Sound and Vibration Measurement A/S in Denmark. The objective of this project was to evaluate the Bruel \& Kjaer sound transmission test equipment with respect to the ASTM E2611-09 standard procedure. All of the work was carried out on thermoplastics and thermoplastic compounds.

\section{STL, Procedure and Apparatus}

\subsection{Sound transmission properties}

The sound transmission coefficient, $\tau$, of a material in a specified frequency band is defined as the fraction of air born sound power incident on a material that is transmitted by the material and radiated on the opposite side expressed as follows,

$$
\tau=W_{t} / W_{i}
$$

Where: $W_{t}$ and $W_{i}$ are the transmitted and incident sound powers respectively

The sound transmission loss, STL, of a material in a specified frequency band is measured in decibels and is ten times the common logarithm of the reciprocal of the sound transmission coefficient i.e.

$$
S T L=10_{\log 10}\left(W_{t} / W_{i}\right)=10_{\log 10}(1 / \tau) d B
$$

\subsection{Procedure and apparatus}

The ASTM E2611-09 standard test procedure for measuring an STL utilises a transfer matrix method for the calculation The data for the calculations are obtained from the sound pressure measurements from four microphones, two positioned between the sound source and the specimen and two positioned on the opposing side of the specimen. The method specifies the geometry of the testing apparatus required with respect to the specimen size, the positioning of the microphones and the measured frequency range. Sound tube testing equipment that conforms to the requirements of E2611-09 is currently being manufactured by Bruel \& Kjaer (B\&K) Sound and Vibration Measurement A/S in Denmark. They can offer two sizes of tube, one with an internal diameter of $100 \mathrm{~mm}$ and the other with an internal diameter of $29 \mathrm{~mm}$. The measured frequency ranges for the tubes are $50 \mathrm{~Hz}-1.6 \mathrm{kHz}$ for the larger tube and $500 \mathrm{~Hz}-6.4 \mathrm{kHz}$ for the smaller tube. A schematic diagram of the four microphone test tube apparatus is shown in figure 1. 


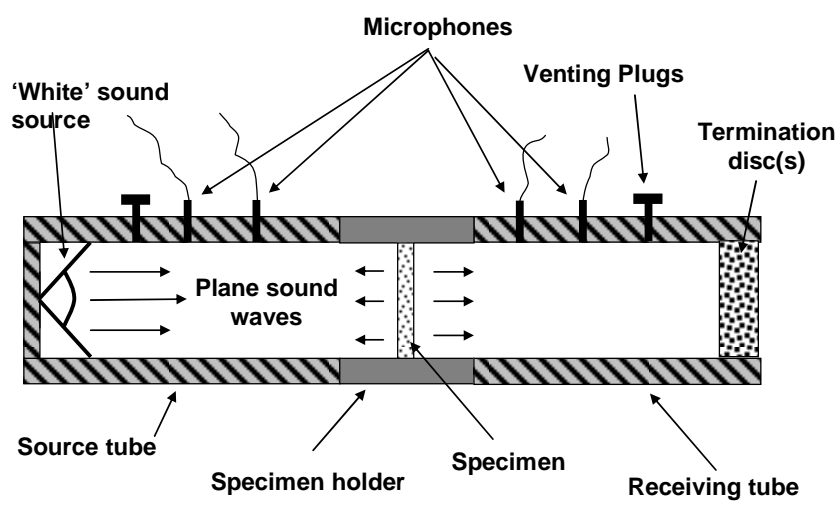

Figure 1. Sound transmission tube assembly. The diagram shows the arrangement for the first of two frequency scans that has one foam termination disc fitted to the end of the receiving tube. The second scan is carried out with two termination discs fitted.

Photographs of the B \& K type 4206-T four microphone impedance tube test equipment that was used in the project are shown in figure 2.

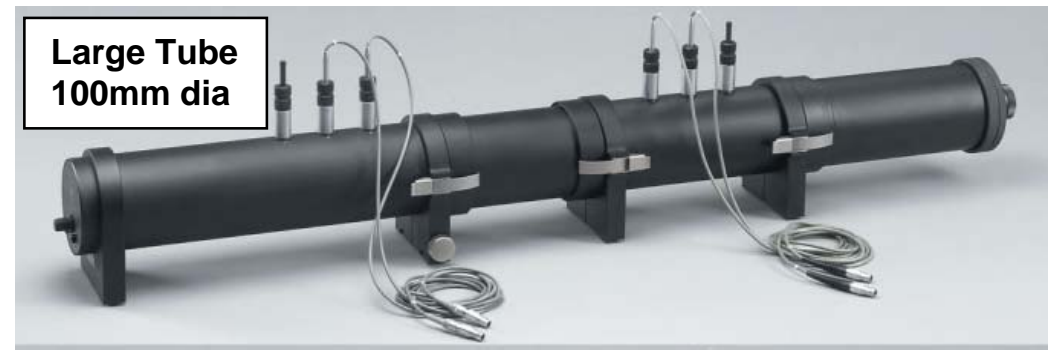

(a)

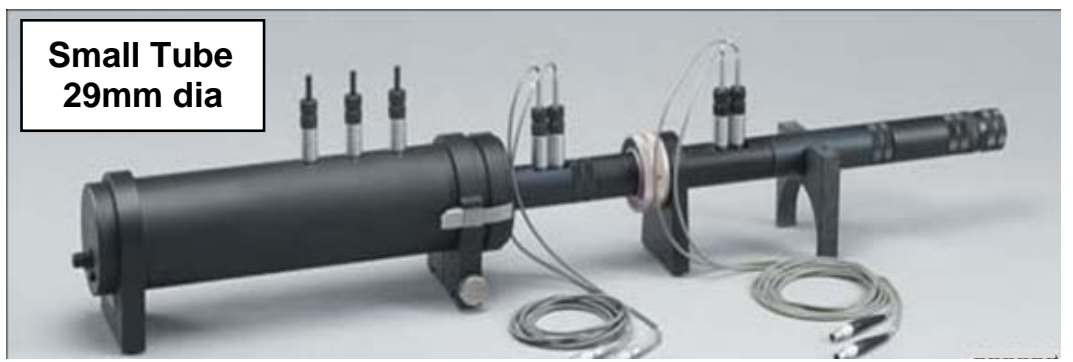

(b)

Figure 2 Bruel \& Kjaer acoustic test tubes showing the configuration for transmission testing. The $100 \mathrm{~mm}$ diameter tube (a) has a frequency range of $50 \mathrm{~Hz}$ to $1.6 \mathrm{kHZ}$. The $29 \mathrm{~mm}$ diameter tube (b) has a frequency range of $500 \mathrm{~Hz}$ to $6.4 \mathrm{kHz}$.

The four microphones in the fixed locations (two in the source tube and two in the receiving tube) record the sound pressures during the test. These are connected to a four channel digital frequency analyser that is used to calculate the complex transfer function from the readings from which it is able to determine the STL of the sample. The results can be displayed as an STL $v$ frequency plot for the range of the 
tube size. The venting plugs shown in the diagram correspond to the third position at each end of the large diameter tube shown in the photograph of figure $2 \mathrm{a}$. The plugs were removed while the sample tube with the sample was being fitted and were replaced for the test. This prevented the sample from being displaced by any compression of the air created by the fitting of the sample tube. This feature was not available on the smaller tube but it was not found to be necessary due to the different clamping method for this assembly.

\section{Sample Preparation and Mounting}

Procedure E2611-09 states that the mounting conditions will 'strongly affect the measured transmission loss'. It also states that the specimen may be 'rigidly mounted or clamped to the wall of the tube, freely suspended with a dense flexible seal or some other mounting method' and that 'care must be taken to mount multiple samples in a consistent manner and to report the mounting method sufficient to reproduce the mount'. Taking these requirements into consideration it was decided initially to investigate the effect of the specimen mounting conditions on the recorded STL measurements and to identify a consistent procedure for this.

The B\&K sound tubes are designed for samples that are fitted flush with the wall of the specimen holder. Consequently the samples could only comply with the freely suspended arrangement as specified in the standard. Figure 3 is a diagram of the mounting condition that we believed would be able to be reproduced without difficulty. The diagram shows a cross section of the specimen holder and the sample. It shows the sample with a thin layer of sealing material between the sample and the holder around the whole circumference and thus there is no direct contact between the specimen and the wall of the holder. This arrangement should enable an accurate measurement of the STL provided that the thickness of the sealing film can be reproduced for different specimens.

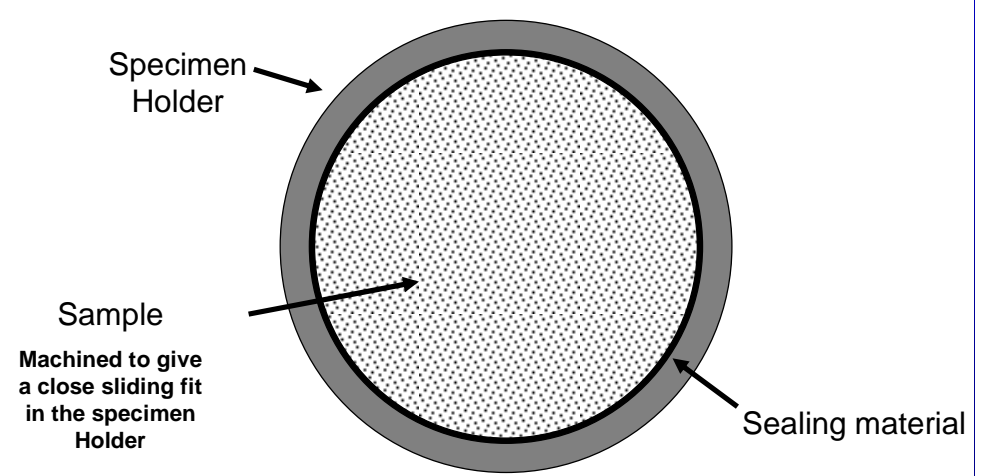

Figure 3. Schematic diagram of the cross section of the sample tube with the sample.

The objective was, therefore, to achieve a machined fit of the sample that just cleared the wall of the specimen holder that would enable a thin layer of sealant to be inserted between the sample and the tube wall.

Test sample discs to suit each of the test tubes (29mm and $100 \mathrm{~mm}$ diameter) were cut from sheets of polypropylene and $30 \%$ glass fibre reinforced polypropylene and were machined on a lathe to the required diameter. The fit of each disc was then tested in the sound tube sample holder. For this a slight contact with the tube wall was required. The sample was then removed and the edge smoothed with fine 
emery paper before the fit in the sample holder was again tested. This procedure was repeated until the sample was able to just slide down the tube under its own weight with the tube in the vertical position.

When the fit of the sample had been achieved, it was mounted into the tube as follows.

Stage 1. A sample positioning cylinder was placed in the specimen holder with spacing blocks inserted at the base of the holder as shown in figure 4 . The length of the positioning cylinder enables the correct distance between the sound source and the front face of the sample to be achieved for every test. The spacer blocks were made to be approximately twice the thickness of the sample for the reason that will become apparent in the next stage of the mounting procedure. The edge of the sample is wetted with the chosen sealing material and the sample is placed in the holder on top of the positioning cylinder. More sealant is then applied to the top surface of the sample around the circumference on the inside of the specimen holder.

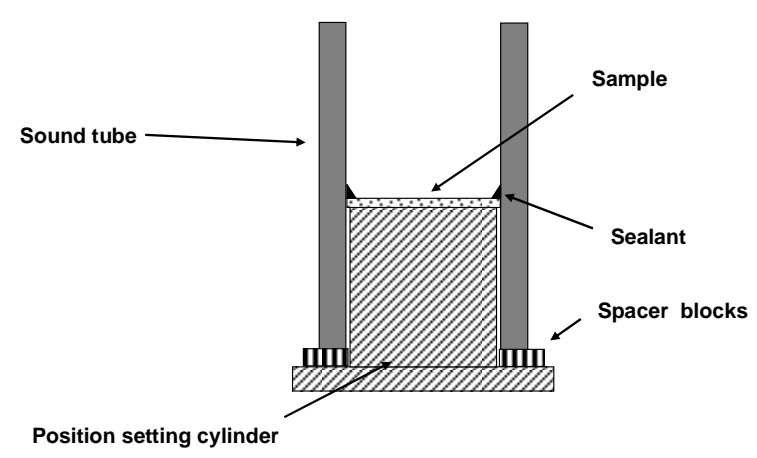

Figure 4. Stage 1 of the sample mounting procedure.

Stage 2. The spacer blocks are removed and the specimen holder is lowered down passed the sample as shown in figure 5 , which causes the sealing material to be pulled down passed the the sample to fill the space between the sample and the holder as shown in figure 3.Excess sealant is then removed from the surface of the sample and the inside of the specimen holder.

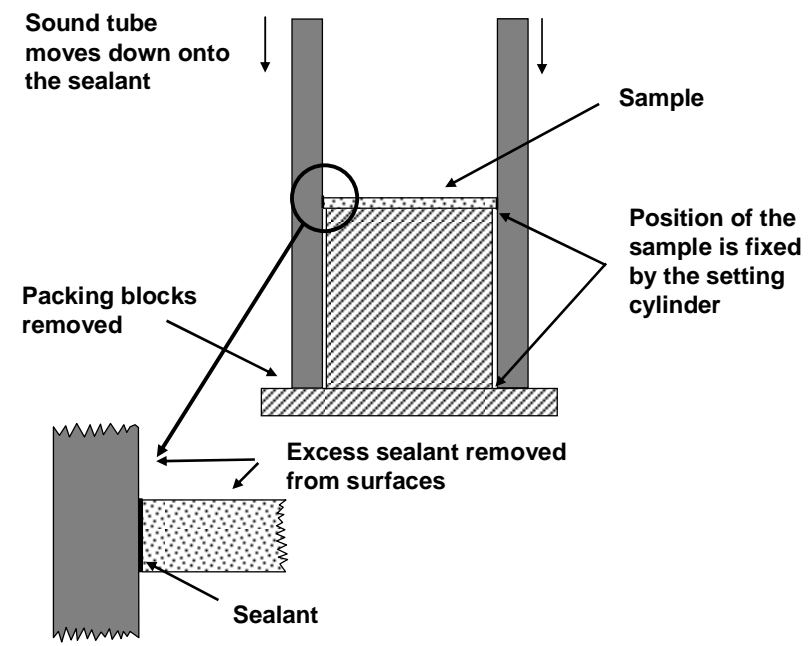

Figure 5 . Stage 2 of the sample mounting procedure 
3. The whole assembly is carefully rotated into the horizontal position and the positioning cylinder removed.

4. The sample holder with the sample is clamped to the source and receiving tube sections with the surface of the sample that was on the positioning cylinder facing the sound source as shown in figure 6 (the venting plugs in the larger tube were removed for the clamping of the sample as explained above)

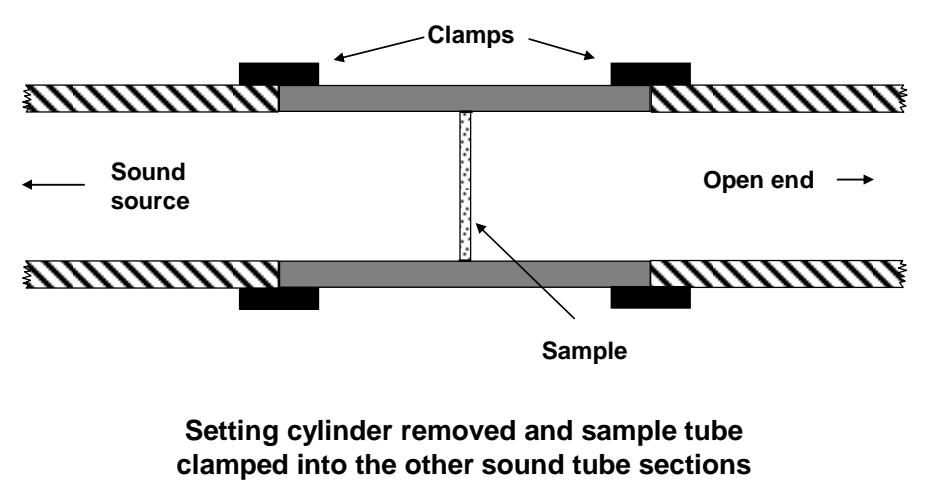

Figure 6 . Stage 4 of the sample mounting procedure.

\section{STL Tests}

Each STL measurement is calculated from two frequency scans on the sample. Figure 1 shows the arrangement for the first scan which is carried out with a standard foam termination disc supplied by B\&K in place. The second scan is carried out with two foam termination discs in place. This procedure enables the analyser to eliminate any extraneous noise signals from the STL calculation. For each sample at least three STL measurements were taken. Each of these measurements was taken following the sample mounting procedure given in the previous section. If the results from the three measurements were not consistent, further measurements were made following repeated mounting of the sample until a reliable result was obtained.

\section{Results}

\subsection{Effect of the sealant}

A series of STL tests was carried out to evaluate the effect of the mounting conditions on the results. For all of the tests the samples were machine to the fit into the sample holder as described in section 3. The following four sealing conditions were investigated;

1 Soft sealants: The objective of these materials was to prevent the leakage of sound around the sample while allowing only a minimal interaction between sample and the wall of the specimen holder.

2 High viscosity soft sealant: This material was used to identify the effect of the viscosity of a soft sealant on the results of the STL test.

3 Tough sealant: To demonstrate the effect of the sample being in contact with the wall of the holder.

4 No sealant: To demonstrate the effect of a sound leak around the sample. 
The two materials used for these tests were polypropylene (PP) and 30\% glass fibre reinforced polypropylene (30GFRPP). The samples were cut from 5 or $6 \mathrm{~mm}$ thick sheets for both of the sizes of the test tubes. The results from the two tube sizes will be presented separately.

\subsubsection{Results from the $29 \mathrm{~mm}$ tube}

Figure 7 shows STL/frequency plots for samples mounted with the soft sealants, silicon oil (SiOil) and petroleum jelly (PJ) in the small test tube. The same sample was used with each mounting material.

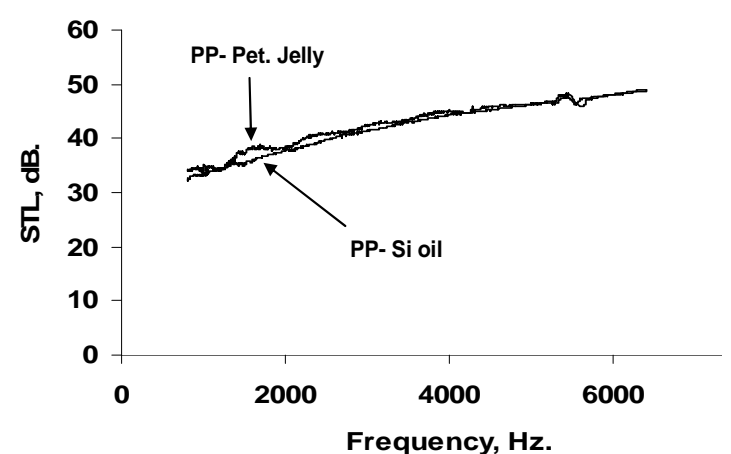

(a)

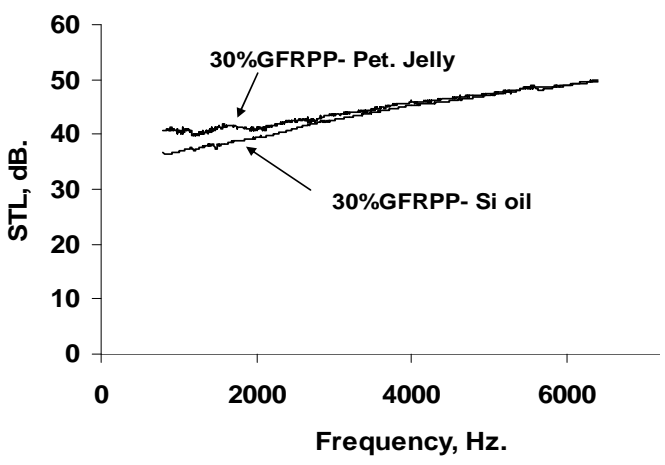

(b)

Figure 7. STL/frequency plots of $6 \mathrm{~mm}$ thick PP (a) and 30\%GFRPP (b) samples sealed with the soft sealants, petroleum jelly and silicon oil.

It can be seen that the samples mounted with silicon oil produced a smoother frequency plot than those mounted with the petroleum jelly. The results also show that the STL for the PJ mounted samples had a slightly greater STL value than the SiOil mounted samples, particularly at the lower end of the frequency range. It is possible that the higher viscosity of the $\mathrm{PJ}$ enabled a slightly greater interaction between the sample and the wall of the holder and thus producing a small increase in the STL. These results suggest that silicon oil is the preferred mounting material for these samples in the small test tube.

Figure 8 shows STL/frequency plots for same samples as those used with the soft sealants mounted with a more rigid sealant. For these samples PTFE tape was wrapped around the edge of the sample before it was inserted into the sample holder. This resulted in a tighter fit for the sample in the holder. 


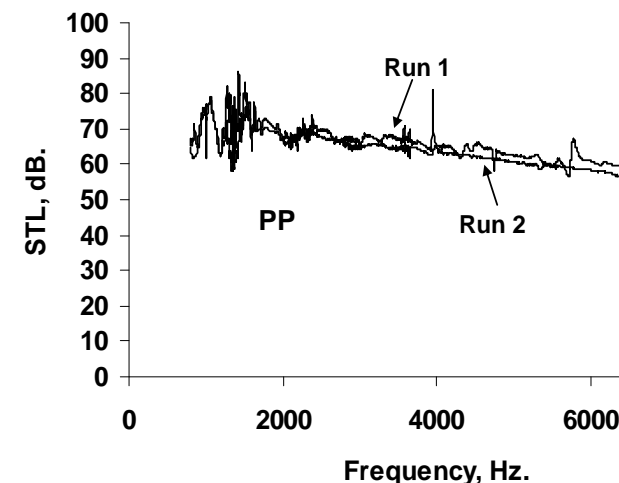

(a)

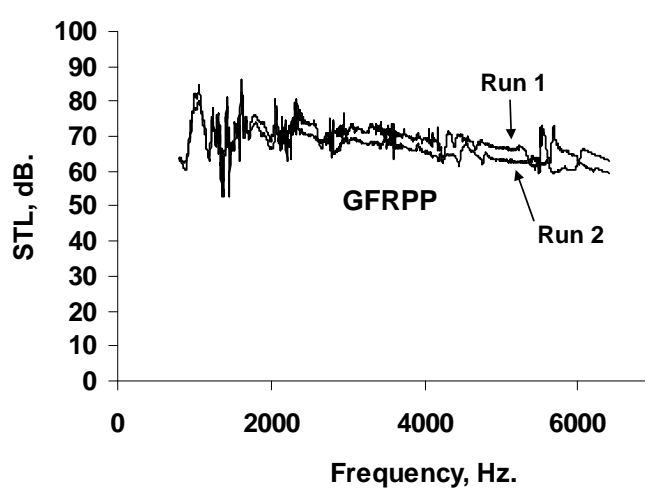

(b)

Figure 8. STL/frequency plots of $6 \mathrm{~mm}$ thick PP (a) and 30\%GFRPP (b) samples sealed with a tough sealant (PTFE tape around the edge of the sample). Two separate tests were carried out on each sample. The samples used were the same as those used for the soft sealing tests of figure 7.

The plots of figure 8 are significantly different from those taken from the identical samples mounted with the soft sealants shown in figure 7 . These plots show typical characteristics to samples that are in contact with the tube wall. In this situation some of the incident sound energy appears to be lost to the tube wall, and in particular at the lower part of the frequency range. Clearly reliable STL data can not be obtained from samples that are in hard contact with the specimen holder wall.

For the last series of tests on these samples they were all tested without any sealant and the results are shown in figure 9.

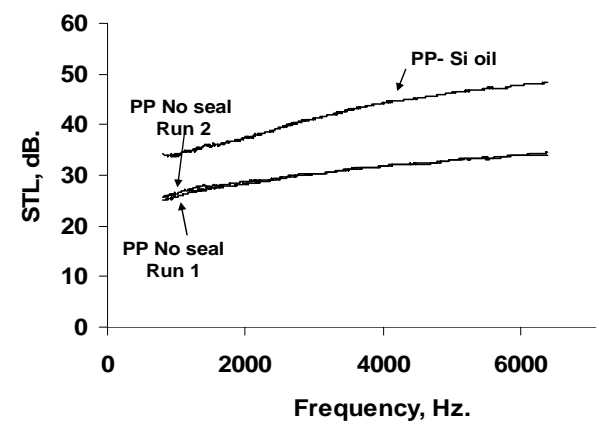

(a)

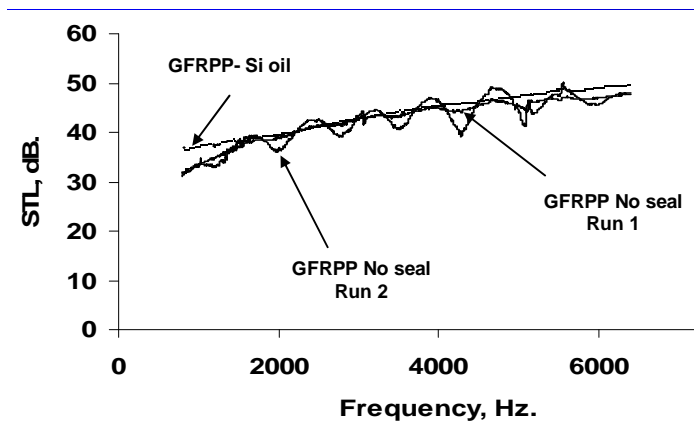

(b)

Figure 9. STL/frequency plots of $6 \mathrm{~mm}$ thick PP (a) and 30\%GFRPP (b) samples sealed with no sealant applied for the test. The results with the silicon oil sealant are shown for comparison. The samples used were the same as those used for the soft sealing tests of figure 7 .

Two measurements were made on each specimen and these are compared to a measurement made on the samples mounted with silicon oil. The GFRPP results from the unsealed samples were close in STL values to the sample mounted with silicon oil but were not consistent and showed an unstable response. The wave pattern, shown particularly in run 2, is indicative of sound leaking around the sample at the tube wall. The results from the PP sample, however, show a very different effect. The traces from each run are very consistent and smooth. A comparison with the plot from the sample mounted with silicon oil shows that the STL of the unsealed sample was significantly lower over the whole frequency range. This result shows that a consistent leakage of sound over the sample was occurring over the whole 
frequency range. The difference between the results from the GFRPP sample and the PP sample could be attributed to the clearance between the samples and the tube wall. The diameters of the two samples were $28.95 \mathrm{~mm}$ and $29.02 \mathrm{~mm}$ for the PP and GFRPP respectively. The PP sample, therefore, had slightly larger clearance at the tube wall that would account for the greater drop in the STL for the unsealed test over the sealed sample than that recorded on the larger GFRPP sample. This result also demonstrates that the soft sealing agent can overcome a slight variation in the clearance between sample and the tube wall.

\subsubsection{Results from the $100 \mathrm{~mm}$ tube}

Figure 10 shows the shows the STL traces for a $6 \mathrm{~mm}$ thick 30GFRPP sample with the four different mounting sealants. The fourth sealant, not used in the tests with the small tube, was a high vacuum grease, HVG, which had a higher viscosity than either the peteroleum jelly or the silicon oil. This material was used to determine the effect of the sealant viscosity on the STL values. The traces shown are the mean values of at least three separate measurements from each of the sealing conditions.

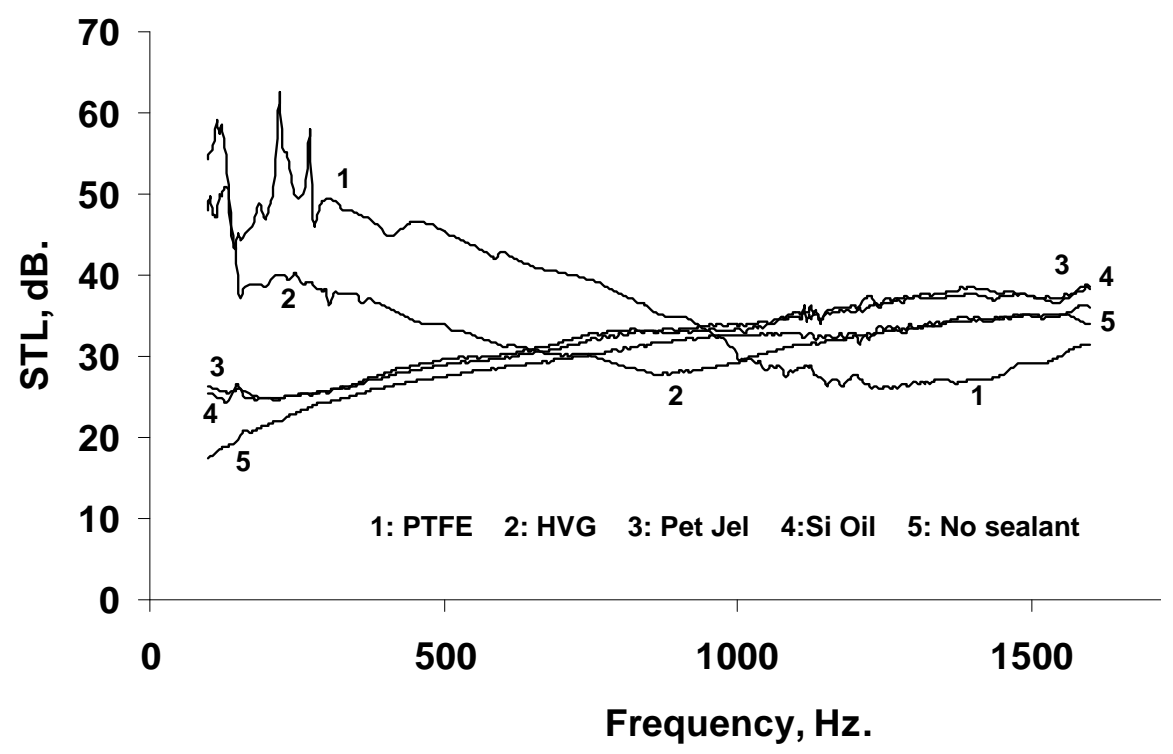

Figure 10. STL/frequency plots of a $6 \mathrm{~mm}$ thick 30\%GFRPP sample from tests with the sample sealed with the four different sealing methods.

The diameter of the sample was measured at $100.01 \mathrm{~mm}$ and the sample was able to slide down the sample holder under its own weight as described in section 3 above. The PTFE 'tight' seal shows that at the low frequencies the contact with the tube wall produces an increase in the STL compared to the samples mounted with the silicon oil and the petroleum jelly. At the higher end of the frequency range, however, the STL shows a decrease compared to these sealants. The high vacuum grease shows a similar, but lesser effect, to the PTFE seal indicating that the viscosity of the grease is high enough to provide an interaction between the sample and the tube wall that affects the STL.

The STL measured without any sealant shows a smooth trace that has a slightly lower value than the traces obtained when the sample was sealed with petroleum jelly or silicon oil. This can be attributed to a slight leakage of sound energy through the small gap between the sample and the wall of the tube. The traces taken when the sample was mounted with peteroleum jelly and silicon oil are almost identical and give confidence that the result is a true value of the STL for that sample. 
Figure 11 shows the STL results for a $6 \mathrm{~mm}$ thick sample of PP using the different sealant materials. The diameter of this sample was $100.03 \mathrm{~mm}$.

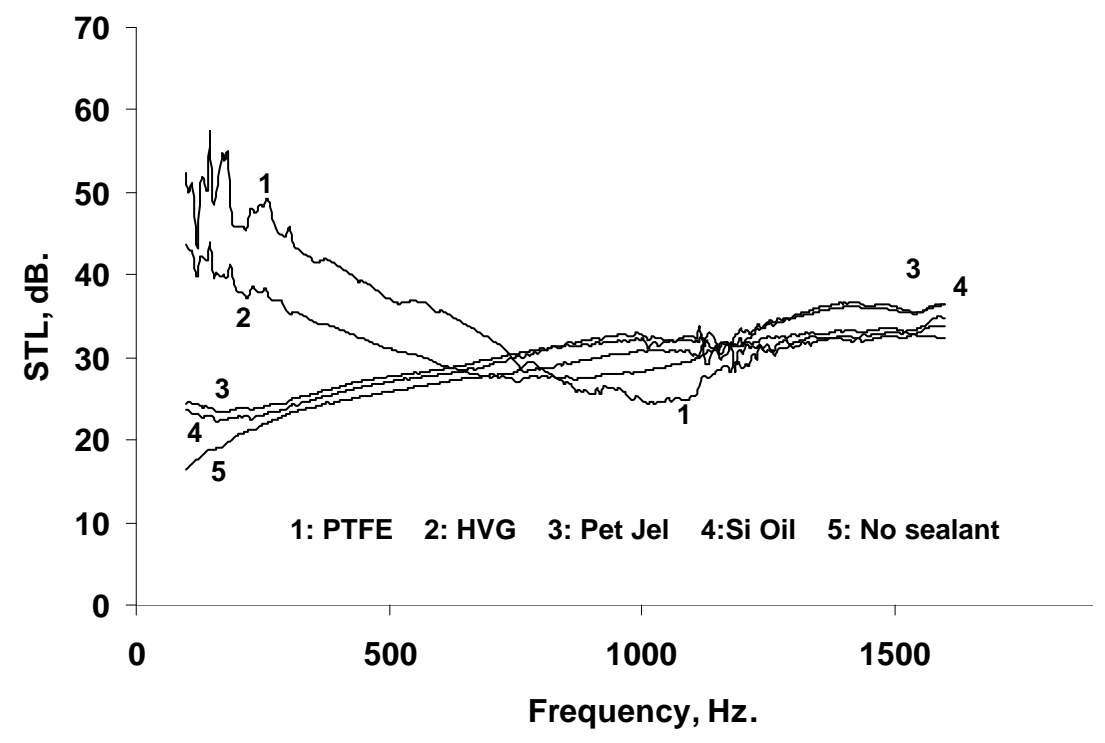

Figure 11. STL/frequency plots of a $6 \mathrm{~mm}$ thick PP sample from tests with the sample sealed with the four different sealing methods.

It can be seen that the trends shown for the GFRPP sample are repeated in these results with the silicon oil and the petroleum jelly providing the most consistent result.

Figures 12 (a) and $12 \mathrm{~b}$ show the STL traces of a $5 \mathrm{~mm}$ thick PP sample taken using the different mounting conditions. The diameter of this sample was $99.92 \mathrm{~mm}$ and this proved to be too loose for a totally unsealed test and a test using silicon oil as a sealant to be carried out because the sample could not be held in position during the test. It was, however, possible to obtain results from these mounting conditions if the sample was lightly pinned at three points of the sample edge with approximately $10 \mathrm{~mm}$ of PTFE tape. The viscosity of the petroleum jelly and the high vacuum grease was sufficient to hold the sample in place during the test.

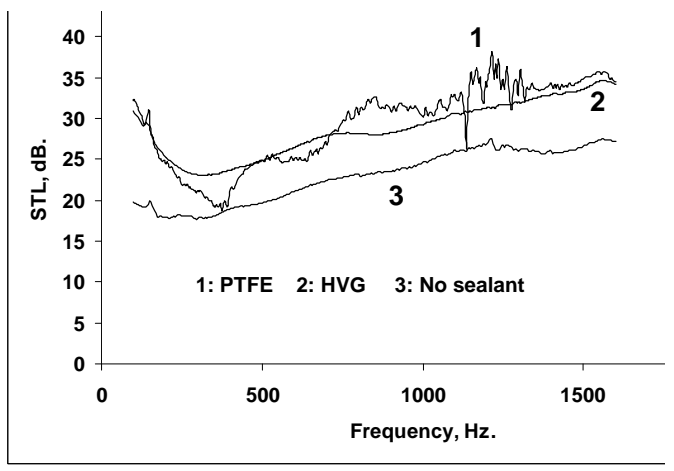

(a)

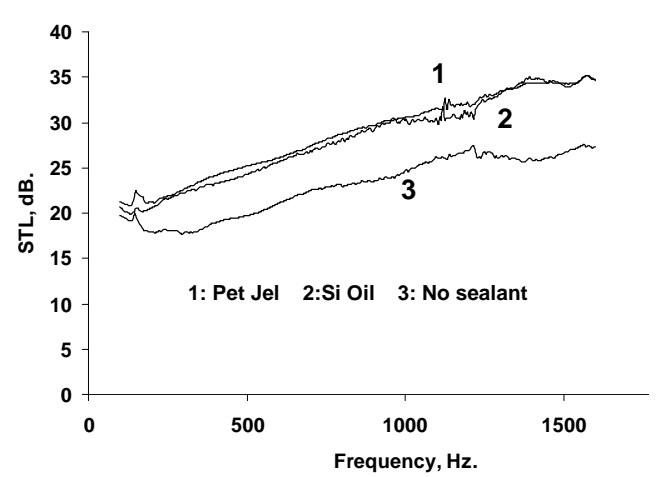

(b)

Figure 12. STL/frequency plots of a $5 \mathrm{~mm}$ thick PP sample from tests with the sample sealed with the four different sealing methods. The trace from the unsealed sample has been put into both (a) and (b) so that a comparison between the other results can be made. 
The trace taken from the unsealed sample is presented in both of the figures so that a comparison between the other results can be made.

The results for this PP sample show a similar trend to those of the GFRPP and the larger $6 \mathrm{~mm}$ PP sample. However, the trace from the unsealed sample shows a greater drop in the STL from the sealed sample than that recorded in the samples with the smaller clearance at the tube wall. This is the same effect that was observed in the results from the $29 \mathrm{~mm}$ test tube and can be attributed to the increased leakage of the sound energy through the larger clearance at the tube wall.

A comparison between the results from the two tube sizes shows that the effects of the sealing conditions for the samples are similar and thus the conditions for the mounting and sealing the samples applies equally to both of the test tubes.

The conclusions from the results on the mounting conditions are:

- A close sliding fit for the sample in the holder can be effectively reproduced.

- A low viscosity sealant produces very consistent STL data when used with polymer based specimens.

- The results presented here show that the sample mounting conditions are critical for obtaining reliable STL data. Characteristics of the STL traces indicative of an interaction between the sample and the tube wall and the occurrence of sound energy leaking around the sample have been demonstrated. Recognition of these characteristics has enabled the identification of soft sealing agents that can give reliable STL data.

\subsubsection{The effect of the incident sound power level}

The intensity of the incident sound waves on the sample can be set prior to each test. The gain control on the instrument amplifier had three settings, $0 \mathrm{~dB}, 6 \mathrm{~dB}$ and $12 \mathrm{~dB}$. All of the results presented in this report were made with the base sound level of $130 \mathrm{~dB}$ and the gain set at $12 \mathrm{~dB}$ giving the value of the incident sound level at $142 \mathrm{~dB}$. However, a series of tests were carried out to investigate the effect of three levels of the incident sound on the STL of samples of PP and 30\%GFRPP. The tests were carried out on the $100 \mathrm{~mm}$ tube with sound levels of 130, 136 and $142 \mathrm{~dB}$. Each of the samples was tested at the three sound levels when mounted with silicon oil, petroleum jelly, high vacuum grease and PTFE tape as described in the previous sections.

The results showed that there was no significant effect of the change in power level on the samples mounted with the 'harder' sealants (HVG and PTFE). The results from the 'soft' sealants showed that there was no significant difference in the STL at the three power levels for the frequency range from approximately $500 \mathrm{~Hz}$ to $1.6 \mathrm{Kz}$. At the lower end of the frequency range, however, there was an increase in the STL as the sound pressure level was decreased. This effect was more pronounced when the samples were mounted with the petroleum jelly than with the silicon oil. Figure 13 shows the results from the samples mounted with petroleum jelly. 


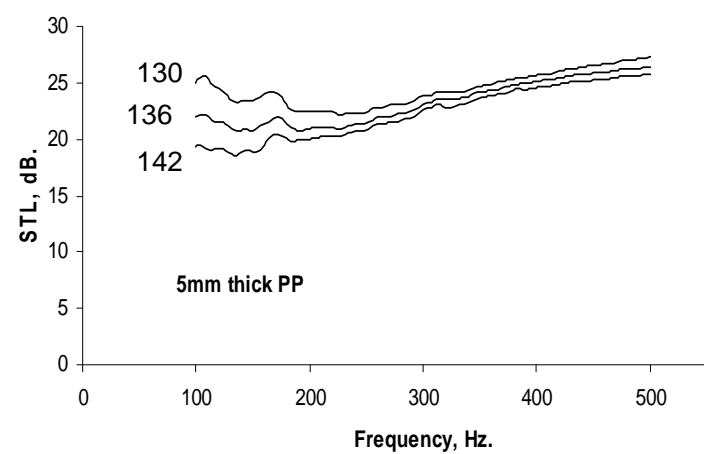

(a)

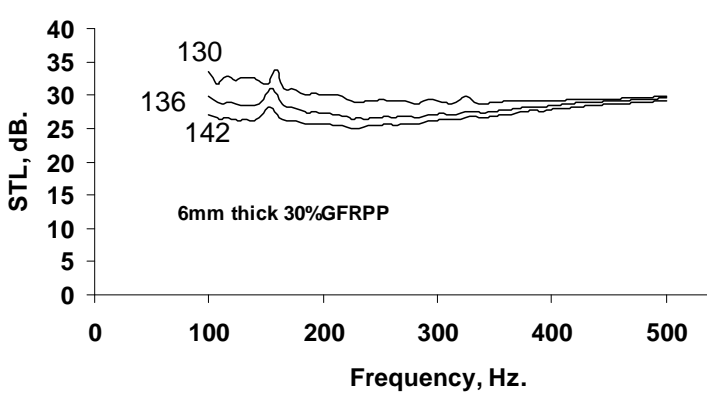

(b)

Figure 13. STL/Frequency plots of (a) a $5 \mathrm{~mm}$ thick PP sample and, (b) a $6 \mathrm{~mm}$ thick $30 \%$ GFRPP sample mounted with petroleum jelly and tested with incident sound levels of 130, 136 and $142 \mathrm{~dB}$.

From these results it can be concluded that the acoustic property of the two samples were linear in the frequency range of $400 \mathrm{~Hz}-1.6 \mathrm{kHz}$. The frequency range up to approximately $400 \mathrm{~Hz}$ shows a slight deviation from linearity. At this stage it is not possible to give an explanation of this result as the method of mounting the sample also appeared to affect the results. This will be a subject of a further investigation.

The result does, however, indicate that the level of the incident sound can affect the STL of a material and that this must be taken into consideration when testing a material for a specific application.

\subsubsection{The continuity between the test tubes}

The sound transmission property of materials can be measured by the sound tube method in the sound frequency range of $0.1-6.4 \mathrm{kHz}$. In order to obtain data for a material over the whole frequency range both of the sound tubes must be used. As stated above, the frequency range for the large tube is $0.1 \mathrm{kHz}-1.6 \mathrm{kHz}$ and the range for the small tube is $0.8 \mathrm{kHz}-6.4 \mathrm{kHz}$. Consequently, there is an overlapping frequency range between the two tubes of $0.8 \mathrm{kHz}$ to $1.6 \mathrm{kHz}$. It was important, therefore, in the assessment of the sample preparation and mounting, to check the results for continuity between the two tubes.

Figure 14 shows the results in the overlapping frequency range for the PP and a PP composite with $70 \%$ w of silicon carbide powder.

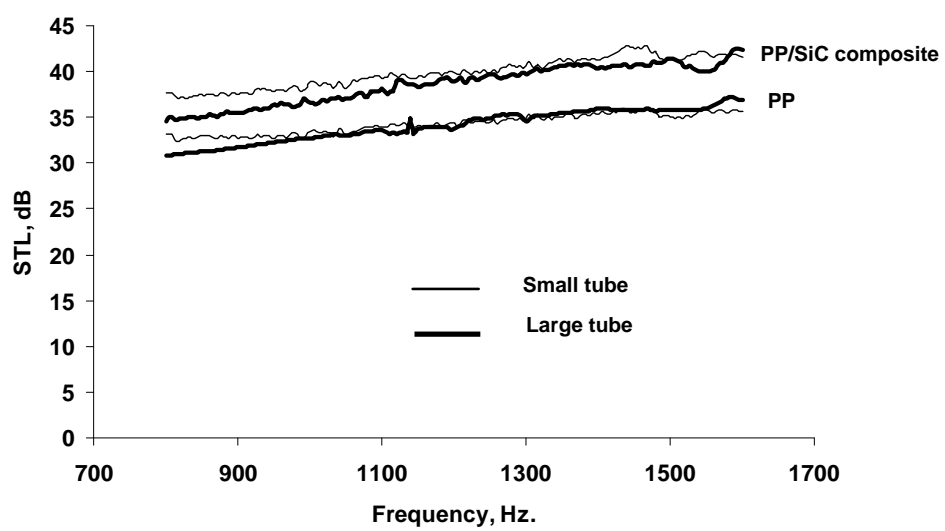


Figure 14. STL values of PP and a composite of PP with 70\%w of silicon carbide filler particles. The plots show the results from the overlapping frequency range between the two sizes of test tube.

The result shows an excellent correlation between the two tubes. This is also an indication that the sample setting and preparation method is consistent and thus gives confidence in the results from both of the tubes.

\subsection{STL of polymers and polymer composites}

The test procedure described in the previous sections was used to carry out a preliminary investigation of the sound transmission properties of a selection of polymers and polymer compounds.

\subsubsection{Effect of sample thickness}

Figure 15 shows the STL/frequency plots of eleven samples of the same high density polyethylene with thicknesses from $1 \mathrm{~mm}$ to $45 \mathrm{~mm}$ in the $100 \mathrm{~mm}$ sound tube. The samples up to $10 \mathrm{~mm}$ thick were taken from solid sheets of the material. The sample thicknesses above $10 \mathrm{~mm}$ were obtained by stacking up to four discs of $10 \mathrm{~mm}$ thick with the addition of a $5 \mathrm{~mm}$ thick disc to give the $15,25,35$, and $45 \mathrm{~mm}$ thick samples. The stacks were made by coating the inner surfaces of the discs with a layer of petroleum jelly before they were pressed together. The samples with thicknesses greater than $10 \mathrm{~mm}$ were not, therefore truly representative of single layer samples of the material but were adequate for an initial evaluation of the effect of thickness on the STL.

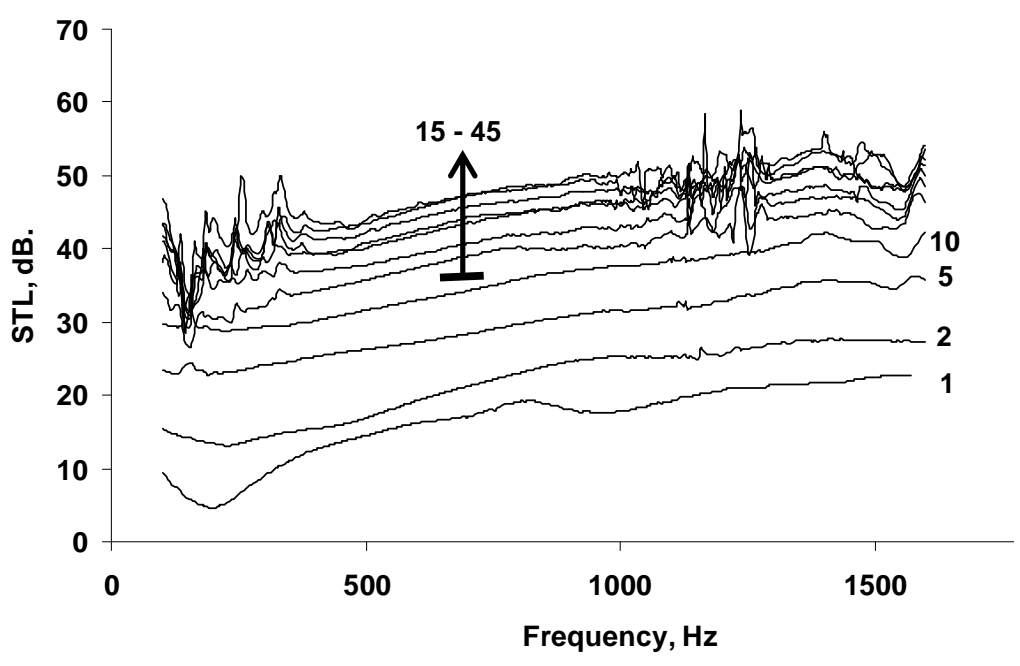

Figure 15. STL/frequency traces of an HDPE resin for sample thicknesses from $1 \mathrm{~mm}$ to $45 \mathrm{~mm}$. The numbers are the sample thickness in $\mathrm{mm}$.

The results show that, as expected, the STL increases with the increase in the section thickness. It also shows that the STL increases with an increase in the sound frequency, an effect that was shown for all of the samples tested in this project.

It can be seen that there are two sections of the frequency range that produced an erratic STL response to the incident sound. The reason for this has not been determined but it is noted that this instability is only present in the samples that were 
made by stacking discs of 10 and $5 \mathrm{~mm}$ and it is more pronounced for the thicker stacks. Thus the joints in the samples may be the cause of the erratic traces. This effect will be investigated in future studies.

From the data that produced the results in figure 15, the values of the STL from the different thicknesses at the frequencies 500,1000 and $1500 \mathrm{~Hz}$. are shown in figure 16. It can be seen that the STL follows a non linear relationship with the sample thickness over the whole frequency range. The results also show that for the HDPE the STL increases sharply up to a sample thickness of about 10 to $15 \mathrm{~mm}$ after which the increase in thickness has a lesser effect on the STL.

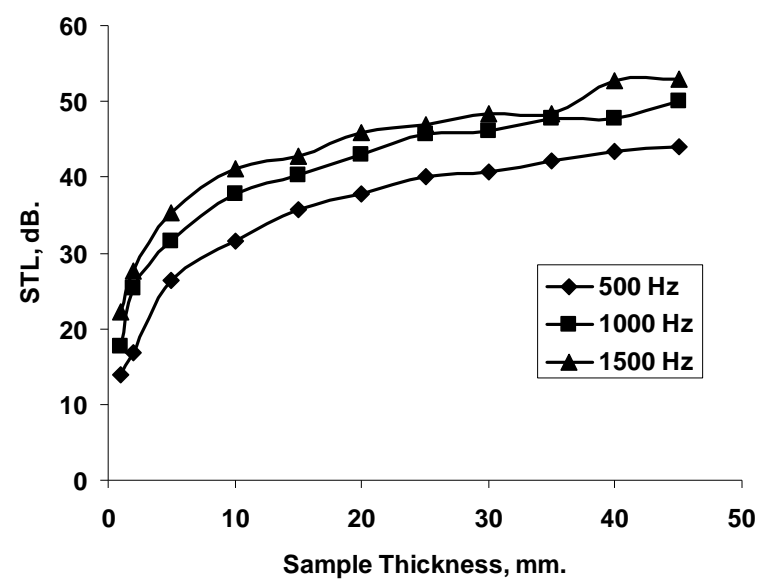

Figure 16. Plots of the STL values at 500,1000 and $1500 \mathrm{~Hz}$ of the HDPE samples against the sample thickness.

In order to obtain a general overview of the STL of a sample over a selected part of the scanned frequency range the average value of the STL over that section of the range can be evaluated. Figure 17 shows the mean STL values of the HDPE samples featured in figure 15 for the frequency range of $100 \mathrm{~Hz}$ to $1.6 \mathrm{kHz}$. This is a convenient way of reporting the data from the STL/ frequency traces that would enable the sound transmission performance of a material to be readily assessed over the range of frequency that a component made from the material would encounter in service. As expected the curve has a similar form to those from the three selected frequencies shown in figure 16.

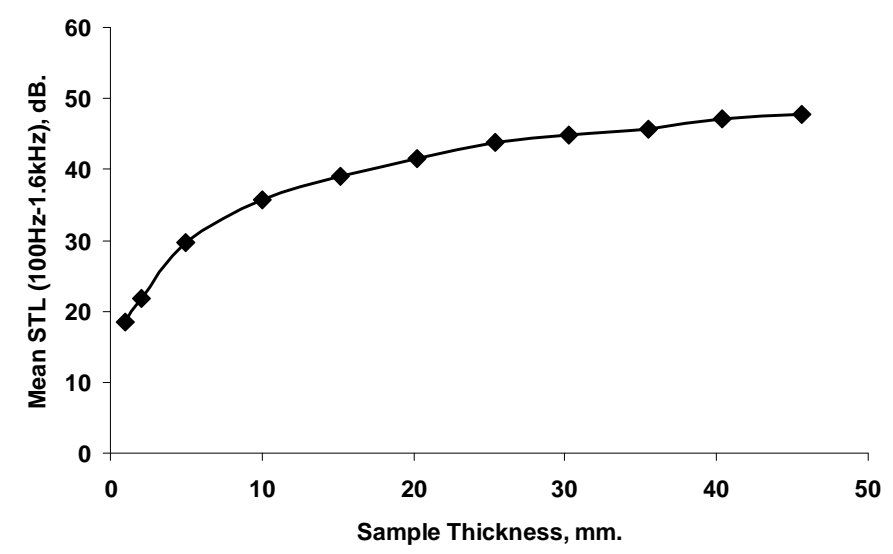

Figure 17. Plot of the mean STL values over the frequency range of $0.1 \mathrm{kHz}$ to 1.6 $\mathrm{kHz}$ of the HDPE samples featured in figure 14 against the sample thickness. 


\subsubsection{Effect of Density and Elastic Modulus}

Figure18 shows the STL/frequency traces of a high density polyethylene, a polypropylene homopolymer and an unplasticised PVC. All of the samples were $5 \mathrm{~mm}$ thick. The difference in the sound transmission performance of these samples must be due to one or more of the following properties; density, modulus and additives. The table shows the mean STL values taken over the whole frequency range $(100 \mathrm{~Hz}-1.6 \mathrm{kHz}$.) of the PVC, PP and HDPE materials compared with the density and tensile modulus values.

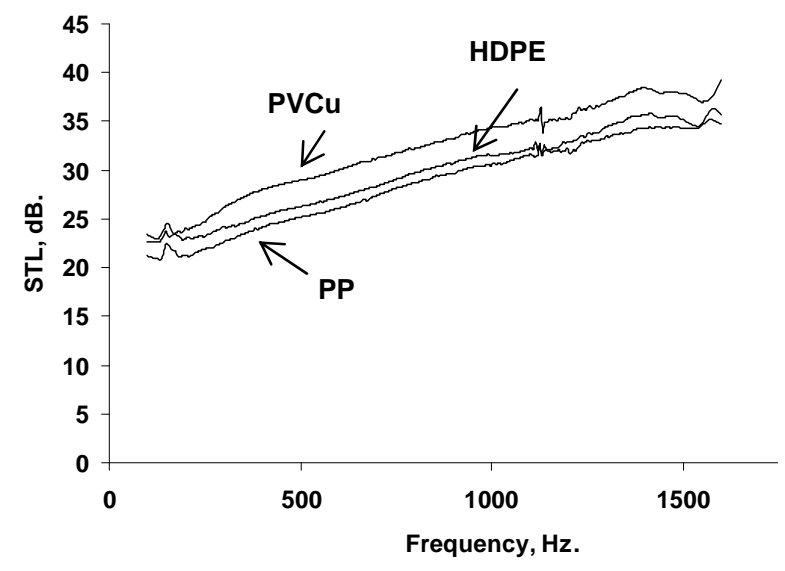

Figure 18. STL/frequency plots of 5mm samples of PP, HDPE and PVCu.

\begin{tabular}{|c|c|c|c|}
\hline Material & $\begin{array}{c}\text { Mean STL } \\
\text { for 0.1- 1.6 } \\
\text { kHz., dB. }\end{array}$ & $\begin{array}{c}\text { Density } \\
\text { g/cc }\end{array}$ & $\begin{array}{c}\text { Tensile } \\
\text { Modulus } \\
\text { GPa }\end{array}$ \\
\hline PP & 28.6 & 0.91 & 1.65 \\
\hline HDPE & 29.7 & 0.96 & 1.00 \\
\hline PVCu & 32.7 & 1.44 & 3.00 \\
\hline
\end{tabular}

Table. Mean STL, tensile modulus and density values of the PP, HDPE and PVCu samples

These results show that the increase in density of the material corresponds to an increase in the STL, a relationship that is not followed by the tensile modulus values. Consequently it appears that density is more significant than tensile modulus for the STL of a material. This result, however, needs to be investigated further before a general conclusion can be made.

\subsubsection{The effect of fillers}

Figure 19 shows the STL/frequency traces from the $29 \mathrm{~mm}$ sound tube of $6 \mathrm{~mm}$ thick samples of the following polypropylene compounds; PP/0v\% filler, PP/13.1v\% Glass fibre, $\mathrm{PP} / 21.8 \mathrm{v} \%$ Bio Ash and $\mathrm{pp} / 33.5 \mathrm{v} \%$ silicon carbide powder. The bio ash was a fine powder that was obtained from the burning of a biomass waste material. 


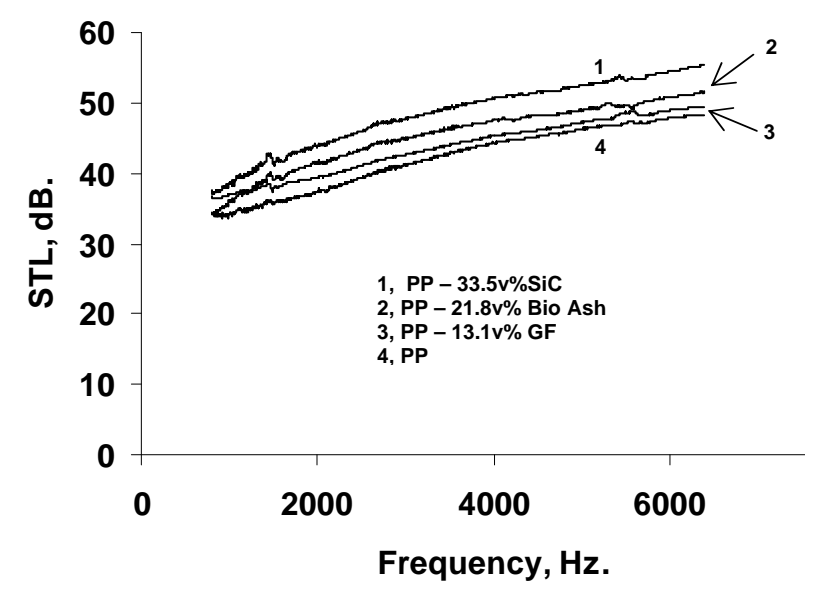

Figure 19. STL/frequency traces of $6 \mathrm{~mm}$ thick samples of compounds with a PP matrix.

The results show that the STL of the compounds increases with the increase in the volume fraction of the filler.

Figure 20 shows a plot of the mean STL values of the samples over the whole frequency range, $800 \mathrm{~Hz}-6.4 \mathrm{kHz}$ against the density of each compound. It can be seen that this relationship is approximately linear. This result supports the results described in section 5.2.2 and shows that the density is a significant factor that will affect the sound transmission property of materials.

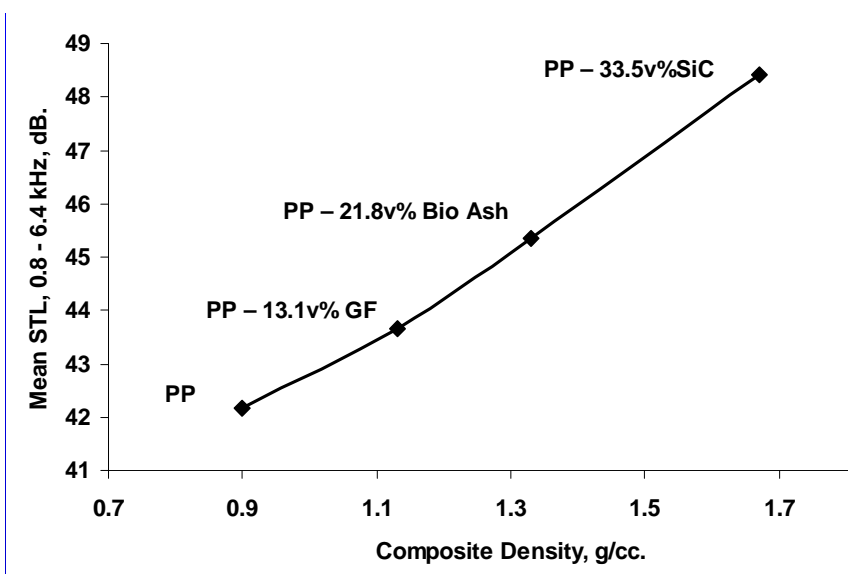

Figure 20. Plot of the mean STL values from the frequency range 0.8 to $6.4 \mathrm{kHz}$ for filled compounds of PP against the density of the compounds

Figure 21 shows a plot of the mean STL over the whole frequency range of the PP composites against the volume fraction of the fillers. 


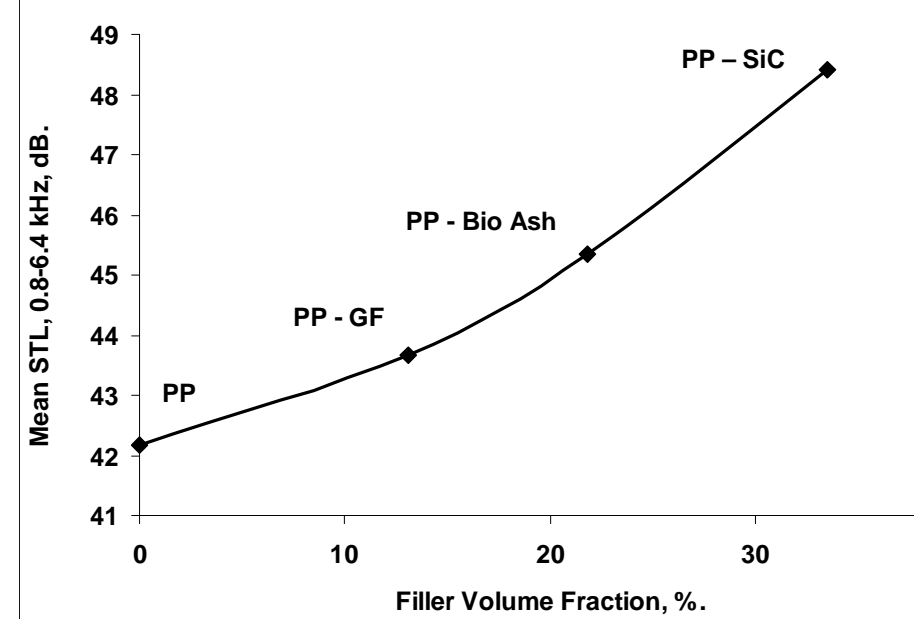

Figure 21. Plot of the mean STL values from the frequency range 0.8 to $6.4 \mathrm{kHz}$ for filled compounds of PP against the volume fraction of the filler in each compound

This result shows a slight deviation from a linear relation. A possible explanation for this effect could be connected to the densities of the fillers which are $2.55 \mathrm{~g} / \mathrm{cc}$, $2.9 \mathrm{~g} / \mathrm{cc}$ and $3.21 \mathrm{~g} / \mathrm{cc}$ for the glass fibre the bio ash and the silicon carbide respectively. If density is a significant property affecting the STL as has been shown in figure 20 and in the table, the glass composite and the bio ash composite will produce a lowering of the plot shown in figure 21 compared to the silicon carbide composite. A more extensive investigation will be required to fully evaluate the relationship between the composite properties and the STL.

\section{Conclusions}

The main conclusions that have been drawn from the results presented are:

- A sound impedance tube with four microphones can be effectively employed to measure the sound transmission loss, STL, of polymers and polymer composites.

- The results of the STL tests are significantly dependant on the sample preparation and mounting procedure. It has been demonstrated that the sample should be machined to give a clearance at the tube wall just sufficient for the sample to have no intimate contact with the wall. If this is then followed up by sealing the clearance gap with a soft/liquid material when it is in position in the test tube, the most consistent and reliable STL data can be achieved.

- Results from tests in which the sample preparation and mounting procedure have deviated from that just given, have demonstrated the effects on the STL results. Thus if any of these effects are observed in a test the cause can be identified.

- It is recommended that at least two tests should be carried out on each sample set up. If the two sets of data are close the mean value should be taken. If there is a significant deviation the sample should be reset and the test run again.

- It has been shown that an increase in the sample thickness is more effective in increasing the STL value when the samples are thin (for the HDPE presented, between $1 \mathrm{~mm}$ and approximately $10 \mathrm{~mm}$ ).

- The STL for all the materials tested showed a gradual increase in the STL value over the whole frequency range covered by the two test tubes.

- The density of a material is a significant factor affecting the STL.

- It has been observed that the incident sound level can have an effect on the STL. It is recommended, therefore, that before a material is assessed for an application, 
the sound intensity level of the intended service conditions are known so that the material can be given a realistic test.

- The sound transmission test tubes provide for a straightforward laboratory method for the evaluation of the STL of materials by a standard test procedure. This would enable materials to be tested and compared before the most promising are put forward to the production of prototype components for full, and expensive, acoustic testing.

- Although there is a standard procedure for the STL test method there is no standard apparatus specified to apply this procedure. The B\&K sound transmission test tubes have been designed to comply with the standard procedure. Thus it would be beneficial for the standard if the B\&K design specification was made as part of the standard.

This initial work has defined a reliable testing procedure for the measurement of the STL of materials using the two sound transmission test tubes. We believe that it forms the foundation for a more detailed study of the sound transmission properties of polymers, polymer composites and materials in general.

\section{Acknowledgments}

The authors wish to thank the Technology Strategy Board (TSB), UK for its financial support of this research and to Bruel \& Kjaer for their technical assistance.

\section{References}

1 - Andrew R. Barnard, Mohan D. Rao, Measurement of Sound Transmission Loss Using a Modified Four Microphone Impedance Tube, NOISE-CON 2004, Baltimore, Maryland (2004).

2 - Peng Dong-Li, Hu Peng, Zhu Bei-Li, 'The modified method of measuring the complex transmission coefficient of multilayer acoustical panel in impedance tube', Applied Acoustics 69, 1240-1248 (2008).

3 - M.J. Mahjoob, N. Mohammadi, S. Malakooti, 'An investigation into the acoustic insulation of triple-layered panels containing Newtonian fluids: Theory and experiment', Applied Acoustics ,70, 165-171(2009).

4 - Olivier Doutres, Yacoubou Salissou, Noureddine Atalla, Raymond Panneton, 'Evaluation of the acoustic and non-acoustic properties of sound absorbing materials using a three-microphone impedance tube', Applied Acoustics, 71, 506-509 (2010).

5 - ASTM E1050-98, 'Standard Test Method for Impedance and Absorption of Acoustical Materials Using a Tube, Two Microphones, and a Digital Frequency Analysis System'. (1998).

6 - ISO 10534-2, 'Determination of sound absorption coefficient and impedance in impedance tubes'. (1998).

7- ASTM E1050-10, 'Standard Test Method for Impedance and Absorption of Acoustical Materials Using a Tube, Two Microphones, and a Digital Frequency Analysis System'. (2010).

8 - ASTM E2611-09, 'Standard test method for the 'Measurement of Normal Incidence Sound Transmission of Acoustical Materials Based on the Transfer Matrix Method", (2009). 
